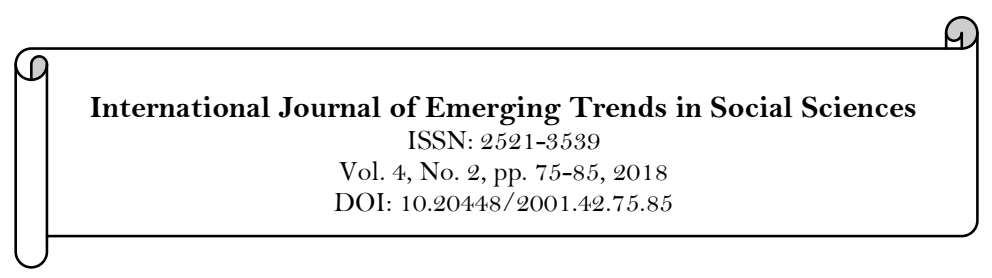

Uapdates

\title{
Coordination Strategy of Dual-Channel Supply Chain for Fresh Product Under the Fresh- Keeping Efforts
}

\author{
Kejing Zhang ${ }^{1 /}$ \\ Jingkai Gao ${ }^{2}$ \\ ${ }^{1,2}$ Glorious Sun School of Business and Management, Donghua University, Shanghai 20005 1, China.
}

\begin{tabular}{|c|c|}
\hline Abstract & \\
\hline $\begin{array}{l}\text { In the context of high loss in the storage and transportation of fresh } \\
\text { agricultural products, in order to help company make reasonable } \\
\text { fresh-keeping decisions and reduce losses, we established a leading } \\
\text { supplier of fresh agricultural products in two level dual channel } \\
\text { supply chain model based on consumer utility function, and using } \\
\text { Stackelberg game method to solve the optimal pricing and optimal } \\
\text { fresh-keeping decision of fresh agricultural supplier and retailer } \\
\text { under centralized decision-making and decentralized decision- } \\
\text { making model. Research shows: (1) Under centralized decision- } \\
\text { making model, the highest profit does not affect the cooperation and } \\
\text { achieve complete coordination regardless of the bargaining power of } \\
\text { the retailer; (2) High cost factor of fresh-keeping efforts makes } \\
\text { supplier and retailer more inclined to lower prices to attract } \\
\text { consumers. (3)The "revenue sharing + fresh-keeping cost sharing" } \\
\text { coordination strategy provided by the supplier can increase the } \\
\text { respective profits of both parties and achieve complete coordination }\end{array}$ & $\begin{array}{l}\text { Keywords: } \\
\text { Dual-channel supply chain } \\
\text { Fresh-keeping efforts } \\
\text { Stackelberg game } \\
\text { Fresh agricultural products } \\
\text { Supply chain coordination } \\
\text { Bargaining power. } \\
\text { Licensed: } \\
\text { This work is licensed under a } \\
\text { Creative Commons Attribution } \\
\text { 4.0 License. } \\
\text { Publisher: } \\
\text { Scientific Publishing Institute }\end{array}$ \\
\hline
\end{tabular}

\section{Introduction}

With the improvement of people's living standard, consumers pay more and more attention to the fresh agricultural product freshness and flavor. In addition, China is a large agricultural country. In the process of transportation and storage, due to insufficient cold storage infrastructure and other fresh-keeping measures, the annual loss of fresh agricultural products has exceeded 300 billion in China. Under this background, this paper studies the fresh-keeping of fresh agricultural products in the supply chain, and puts forward the coordination strategy of node enterprises in fresh agricultural products dual-channel supply chain, which has double significance to improve the efficiency of enterprises and consumer life satisfaction.

Ferguson and Ketzenberg (2006) pointed out that food retailer may cause up to $15 \%$ loss due to damage and corruption of perishable items in Western developed countries. Fresh agricultural products have the characteristics of being corrosive and have a large loss during production and transportation. Martin (2015) pointed out that more than $25 \%$ of fruits and vegetables in China will deteriorate in transportation to wholesale markets and stores. So in this context, many scholars began to study the supply chain of fresh agricultural products. For example, Yu and Nagurney (2013) studied the network-based fresh agricultural product supply chain model, and set product differentiation due to the freshness and corrosion resistance of the product. The variational inequality formula of Cournot-Nash equilibrium condition in food supply chain network is used to indicate that product differentiation may be an effective strategy for enterprises to maintain financial flexibility. Huang, He, and Li (2018) studied the Stackelberg game model of a three-level food supply chain, studied the optimal pricing, inventory decisions and maximize profits of products under decentralized decision making. The results show that the vertical cooperation of supply chain members helps to increase the total profit, and the retailer's protection investment is not only beneficial to itself, but also beneficial to supplier.

The high loss rate caused by the corrosiveness, irreversibility and vulnerability of fresh agricultural products has always been a difficult problem for managers of fresh agricultural products, and also a hot issue in academic circles. Cai, Chen, and Xiao (2010) studied the retailer's supply chain for sourcing a certain amount 
of fresh products from supplier. During the transportation process, retailer must make appropriate efforts to maintain product freshness. In the decentralized and centralized decision-making model, the model is used to study the optimal decision of each node of the supply chain system. Nagurney, Besik, and Yu (2018) studied a competitive food supply chain network model, in which fresh agricultural producers use their supply chain network to produce and distribute the amount of fresh product to maximize their own interests, and consumers respond to each other through the price they are willing to pay. Lee and Dye (2012) assumed that demand was affected by inventory levels, and retailer reduced the rate of deterioration of fresh product through the use of fresh-keeping technology. Based on this, the retailer's replenishment plan and optimal fresh-keeping technology investment were studied. Aviv and Pazgal (2008) studied the pricing strategy of seasonal commodities. Strategic consumers market in a Poisson process and their valuations decrease over time. Under the inventory-related price reduction strategy and the pre-announced price reduction strategy, the subgame perfect Nash equilibrium of the game between enterprises and strategic consumers is analyzed.

In the supply chain coordination of fresh agricultural products, Gu, Fu, and Li (2018) studied a fresh product supply chain consisting of a fresh product supplier and an e-retailer, and derived the optimal price, quantity and fresh-keeping effect by analyzing the model. In the end, it is shown that the rate of return has nothing to do with the loss of freshness and the loss of consumers. The revenue sharing contract cannot achieve channel coordination under the formulation of our model. Tiaojun, Danqin, and Houcai (2011) assumed that consumer utility is affected by product quality, service quality and retail price. On this basis, the retailer's optimal service quality and pricing decision are given. The final revenue sharing contract realizes the supply chain Pareto improvement and coordination. Su and Zhang (2008) studied the impact of strategic customer behavior on supply chain performance and designed multiple contracts to achieve supply chain coordination.

Based on the current research of scholars, in the context of the dual channel of fresh agricultural products, This paper focuses on the characteristics that freshness of fresh agricultural products decreases with time and the cost of fresh-keeping effort invested by suppliers who dominate the supply chain, and construct the utility function influenced by freshness and price, analyzing and comparing the effects of profit and optimal decision-making of supply chain system when fresh produce suppliers put in the cost of fresh-keeping effort. Finally, the two parties have formulated a reasonable fresh-keeping "cost sharing contract + revenue sharing" model, and achieved complete coordination.

\section{Model Symbols and Assumptions 2.1. Model Symbols}

Table-1. Symbol Description.

\begin{tabular}{l|l}
\hline Model symbols & Symbol Description \\
\hline$T$ & Life cycle of fresh product \\
\hline$\lambda(t)$ & Freshness function over time \\
\hline$\lambda_{0}$ & Initial freshness of a fresh product \\
\hline$\theta_{0}$ & Attenuation factor of freshness of fresh product \\
\hline$k$ & Supplier of fresh-keeping effort level \\
\hline$\xi$ & Sensitivity factor of freshness to the fresh-keeping efforts \\
\hline$\mu$ & Effort cost coefficient of fresh-keeping \\
\hline$p$ & Market price of fresh product \\
\hline$\pi$ & Profit of whole supply chain of fresh agricultural products \\
\hline$\pi s$ & Fresh agricultural product supplier's profit \\
\hline$\rho$ & Fresh agricultural product retailer's profit \\
\hline$w$ & Market share of offline retail channels \\
\hline$\eta$ & Retailer's wholesale price \\
\hline$\varphi$ & Fresh-keeping cost sharing ratio \\
\hline$\tau$ & Revenue sharing ratio \\
\hline$\beta$ & Production cost of fresh product \\
\hline & Consumer demand at any one time \\
\hline
\end{tabular}

In addition, the unified regulation, in the superscript of the formula, the letter $c$ is the centralized decision, the letter $d$ is the decentralized decision, the superscript $*$ indicates the coordination model; In the 
subscript of the formula, the subscript contains $S$ for the online channel, and the subscript contains $r$ for the offline channel.

\subsection{Model Assumption}

Assumption 1. As retailer face the whole retail market, retailer have a market advantage terminal, so retailer to adopt a uniform pricing, the dominant supplier and retailer to determine wholesale prices by negotiation.

Assumption 2. The freshness function is $\lambda(t)=\lambda_{0}-\theta_{0}\left(\frac{t}{T}\right)^{\frac{1}{2}}, t \in(0, T)$, that is, the freshness function decreases with time, Where T represents the life cycle of fresh product, from the beginning of sales until the entire sales period of zero the effectiveness of fresh product, $\theta_{0}$ indicates the freshness decay factor of fresh products over time, $\lambda_{0} \mathrm{Y}$ indicates the initial freshness of a fresh product; In addition, the level of effort invested by one party uses the product form to influence the attenuation factor $\theta_{0}$ of fresh product freshness, that is, the freshness function after inputting fresh-keeping efforts is $\lambda(t)=\lambda_{0}-\theta_{0}(1-\xi k)\left(\frac{t}{T}\right)^{\frac{1}{2}}, t \in(0, T)$

Assumption 3. The fresh-keeping effort cost function is $C(k)=\frac{1}{2} \mu k^{2}$, where $\mu$ represents the coefficient of the cost of fresh-keeping effort, and $k$ represents the level of fresh-keeping efforts, and this form of the second-party effort cost is widely used.

Assumption 4. It is assumed that during the entire life cycle of a fresh agricultural product, when the utility of the consumer demand is greater than zero, the purchase behavior will occur. This paper constructs the utility function of the online direct selling channel at a certain time $t$ is $U_{s}(t)=U_{0}-p+\beta \lambda_{s}(t)$, and the utility function of the offline retailer channel at a certain time $\mathrm{t}$ is $U_{r}(t)=U_{0}-p+\beta \lambda_{r}(t), U_{0}$ represents the initial cognitive value of the consumer to a fresh agricultural product, $\beta$ represents The sensitivity of the market to the freshness of a fresh product, They all obey the uniform distribution of $[0,1]$.

Assumption 5. There is only one supplier and one retailer in the entire dual-channel supply chain system, and both parties have rational decision-making and risk neutrality in the case of information sharing, which is in line with the conditions of the Stackelberg game model.

\section{Problem Statement and Model}

\subsection{Problem Statement}

According to the dual-channel supply chain model of fresh agricultural products in real life, a Stackelberg game model is constructed, in which a single supplier of fresh agricultural products is the leader and the retailer of fresh agricultural products is the follower. On the one hand, the retailer facing the market terminal determine the uniform price of fresh agricultural products in the market; on the other hand, the upstream supplier take fresh agricultural products to expand sales volume, and distribute fresh agricultural products to retailer at wholesale prices, and retailer sell them to consumers through the model of physical store operation; Fresh agricultural products supplier have actively expanded the online direct marketing channels through the Internet to sell fresh agricultural products directly through the network channels. The fresh agricultural products in the dual channel supply chain structure as shown in Figure 1. 


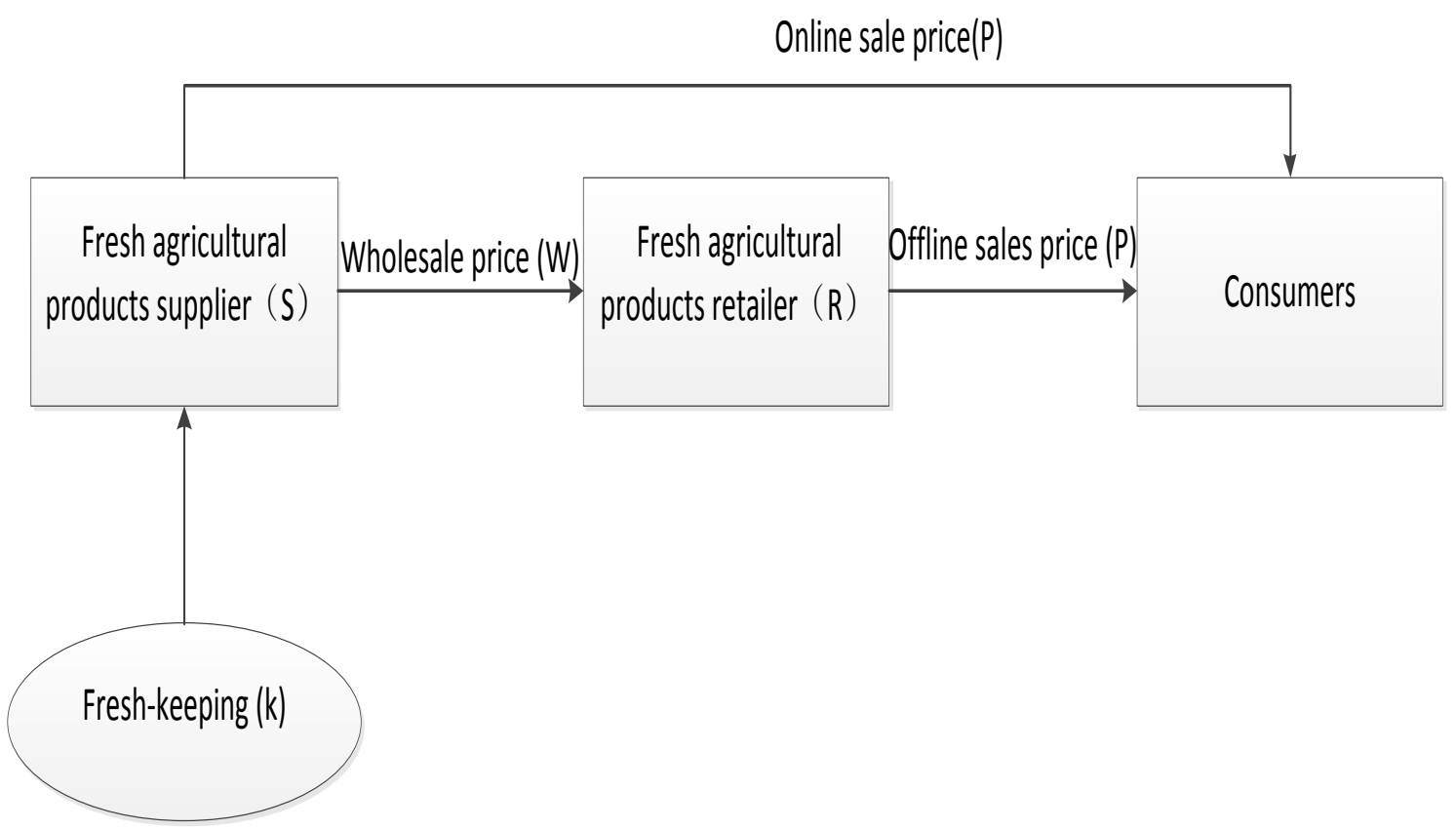

Figure-1. Fresh agricultural products in the dual channel supply chain structure.

\subsection{Centralized Decision Model}

Based on the above assumptions, only when consumers buy that utility is greater than 0 , consumers will choose to buy some fresh agricultural products, the demand at any time under the line:

$$
\begin{gathered}
D_{s}(t)=(1-\rho) \tau P\left(U_{s}(t)>0\right)=(1-\rho) \tau P\left(U_{0}>p-\beta \lambda_{s}(t)\right) \\
D_{r}(t)=\rho \tau P\left(U_{r}(t)>0\right)=\rho \tau P\left(U_{0}>p-\beta \lambda_{r}(t)\right)
\end{gathered}
$$

During the whole life cycle of a fresh agricultural product, the sales volume of fresh agricultural products through the supplier's online channel is as follows:

$$
\begin{aligned}
& D_{s}=\int_{0}^{\mathrm{T}} D_{s}(t) d t=(1-\rho) \tau \int_{0}^{\mathrm{T}} P\left(U_{0}>p-\beta \lambda_{s}(t)\right) d t \\
& =(1-\rho) \tau\left(\frac{1}{3} T\left(3-3 p+2 \beta(-1+k \xi) \theta_{0}+3 \beta \lambda_{0}\right)\right)
\end{aligned}
$$

Similarly, the sales volume of fresh agricultural products under offline channels is:

$$
D_{r}=\int_{0}^{T} D_{r}(t) d t=\rho \tau\left(\frac{1}{3} T\left(3-3 p+2 \beta(-1+k \xi) \theta_{0}+3 \beta \lambda_{0}\right)\right)
$$

Among them, A denotes the demand of fresh agricultural products on-line direct marketing channels; B denotes the demand of fresh agricultural products on-line and off-line retail channels; In addition, $A=\frac{2}{3} T \beta \xi \tau \theta_{0} ; B=T \tau-\frac{2}{3} T \beta \tau \theta_{0}+T \beta \tau \lambda_{0}$.

When the centralized decision of fresh agricultural products supplier and retailer of fresh agricultural product, this is to maximize the overall interests of the supply chain profit as the only goal, the objective function of the whole system at this time:

$$
M A X \pi(p, k)=(p-c) D_{s}+(p-c) D_{r}-C(k)
$$

Theorem 1 In the centralized model, the profit function of the supply chain is a concave function about the fresh-keeping effort and price, and the profit function A has a unique maximum.

Proof: The Hessen matrix of supply chain profit function on price and fresh-keeping efforts is $\left|\begin{array}{cc}-2 T \tau & A \\ A & -\mu\end{array}\right|=2 T \tau \mu-A^{2}>0, \frac{\partial^{2} \pi}{\partial p^{2}}=-2 T \tau<0$, the two order Hesse matrix is negative, theorem 1 proof.

The price and fresh-keeping efforts for a first derivative, $\frac{\partial \pi^{c}}{\partial p}=0, \frac{\partial \pi^{c}}{\partial k}=0$, optimal solution of simultaneous equations was born in dual channel supply chain of fresh agricultural products are as follows: 


$$
\begin{aligned}
p^{c} & =\frac{-A^{2} c+B \mu+c T \mu \tau}{A^{2}-2 T \mu \tau} \\
D_{s}^{c} & =\frac{T \mu \tau(\rho-1)(B-c T \tau)}{A^{2}-2 T \mu \tau} \\
D_{r}^{c} & =\frac{T \mu \rho \tau(c T \tau-B)}{A^{2}-2 T \mu \tau} \\
k^{\mathrm{c}} & =\frac{A(c T \tau-B)}{A^{2}-2 T \mu \tau}
\end{aligned}
$$

Under the centralized decision, the maximum profit of the dual channel supply chain is:

$$
\pi^{c}\left(p^{c}, k^{c}\right)=\frac{\mu(B-c T \tau)^{2}}{2\left(2 T \mu \tau-A^{2}\right)}
$$

\subsection{Decentralized Decision Model}

Under the decentralized decision model, The supplier and retailer of fresh agricultural products play Stackelberg game with the goal of maximizing their own profits, The retailer of fresh agricultural products (R) determines the uniform price of the market according to the judgment of the market and previous data; Finally, the supplier of fresh agricultural products determined the fresh-keeping effort according to the market price and the wholesale price negotiated by both sides. The game process of this decision can be described by the following model:

$$
\begin{aligned}
& \text { MAX } \pi_{s}=(p-c)(1-\rho)(B+A k-T \tau p)+(w-c) \rho(B+A k-T \tau p)-\frac{1}{2} \mu k^{2} \\
& \text { S.T. } \pi_{r}=(p-w) \rho(B+A k-T \tau p)
\end{aligned}
$$

Retailer consider the maximization of their profits to determine the unified price of fresh agricultural products , $\quad \frac{\partial^{2} \pi_{r}}{\partial p^{2}}=-2 T \rho \tau<0 \quad, \quad$ order $\quad \frac{\partial \pi_{r}}{\partial p}=0 \quad, \quad$ The equation of the answer is $p^{d}=\frac{B+A k+T w \tau}{2 T \tau} 。$

Theorem 2 when $\mathrm{A}=0$, the profit of fresh agricultural supplier is a concave function of wholesale price $w$ and the level of fresh-keeping $k$.

Proof: the second-order partial derivative of profit function $\pi_{s}$ to $k$ and wholesale price $p$ is $\frac{\partial^{2} \pi_{s}}{\partial k^{2}}=-\mu+\frac{A^{2}(1-\rho)}{2 T \tau}<0 \quad, \quad$ that $\quad$ is $\quad \mu>\frac{A^{2}(1-\rho)}{2 T \tau} \quad ; \quad \frac{\partial^{2} \pi_{s}}{\partial w^{2}}=-\frac{1}{2} T(1+\rho) \tau<0$, $\frac{\partial^{2} \pi_{s}}{\partial w \partial k}=\frac{\partial^{2} \pi_{s}}{\partial k \partial w}=\frac{A \rho}{2}$, two order, Hesse matrix is greater than zero, namely $-\frac{A^{2}}{4}+\frac{1}{2} T \mu \tau(1+\rho)>0$, that is $\mu>\frac{A^{2}}{2 T \tau(1+\rho)}$, therefore, the solution is $\mu>\frac{A^{2}}{2 T \tau(1+\rho)}$, proof of theorem 2 .

Under decentralized decision-making model, the optimal decision for fresh agricultural products is as follows is:

$$
\begin{gathered}
p^{d}=\frac{A^{2} c-\mu(B+2 B \rho+c T \tau)}{A^{2}-2 T \mu \tau(1+\rho)} \\
w^{d}=\frac{A^{2} c-2 \mu(B \rho+c T \tau)}{A^{2}-2 T \mu \tau(1+\rho)} \\
D_{s}^{d}=\frac{T \mu \tau(1-\rho)(-B+c T \tau)}{A^{2}-2 T \mu(1+\rho) \tau}
\end{gathered}
$$




$$
\begin{aligned}
D_{r}^{d} & =\frac{T \mu \rho \tau(-B+c T \tau)}{A^{2}-2 T \mu(1+\rho) \tau} \\
k^{d} & =\frac{A(-B+c T \tau)}{A^{2}-2 T \mu \tau(1+\rho)}
\end{aligned}
$$

By substituting the optimal decision of supplier and retailer into Formula 11, the profit of fresh product supplier and retailer under decentralized decision is obtained as follows:

$$
\begin{aligned}
\pi_{s}^{d} & =\frac{\mu(B-c T \tau)^{2}}{2\left(2 T \mu \tau(1+\rho)-A^{2}\right)} \\
\pi_{r}^{d} & =\frac{T \mu^{2} \rho \tau(B-c T \tau)^{2}}{\left(A^{2}-2 T \mu \tau(1+\rho)\right)^{2}}
\end{aligned}
$$

Therefore, in the decentralized model, according to the profits of retailer and supplier can draw the total profit of the entire supply chain:

\subsection{Comparison of Two Models}

$$
\pi^{d}=\frac{\mu(B-c T \tau)^{2}\left(-A^{2}+2 T \mu \tau(1+2 \rho)\right)}{2\left(A^{2}-2 T \mu \tau(1+\rho)\right)^{2}}(19)
$$

Proposition $1 \frac{\partial p^{c}}{\partial \mu}<0, \frac{\partial p^{d}}{\partial \mu}<0$ 。

Proof: By the formula 6 obtained $\frac{\partial p^{c}}{\partial \mu}=\frac{A^{2}(c T \tau-B)}{\left(A^{2}-2 T \mu \tau\right)^{2}}$, from formula 10 and formula 9, We know that the overall profit and freshness level of fresh agricultural products supply chain are satisfied: $\pi^{c}>0$, $0<k^{c}<1$, so, we can draw: $A^{2}-2 T \mu \tau<0, c T \tau-B<0$, in summary, $\frac{\partial p^{c}}{\partial \mu}<0$;we can obtain $\frac{\partial p^{d}}{\partial \mu}=-\frac{A^{2}(1+2 \rho)(B-c T \tau)}{\left(A^{2}-2 T \mu \tau(1+\rho)\right)^{2}}<0$ from formula 12, and combining formula 16 and formula 17 , we can conclude that: $B-c T \tau>0$, so $\frac{\partial p^{d}}{\partial \mu}<0$; Proposition 1 is proved.

Proposition 1 shows that under centralized and decentralized decision-making, the optimal price of fresh agricultural products is negatively correlated with the cost coefficient of fresh-keeping, indicating that under these two models, supplier of fresh agricultural products attract consumers through fresh-keeping decisionmaking will bring greater burden to themselves, combined with the timeliness of fresh agricultural products, so the use of low. Price policy attracts consumers to spend.

Proposition $2 \frac{\partial D_{s}^{c}}{\partial \mu}<0, \frac{\partial D_{r}^{c}}{\partial \mu}<0 ; \frac{\partial D_{s}^{d}}{\partial \mu}<0, \frac{\partial D_{s}^{d}}{\partial \mu}<0$

Proof: obviously $\quad B-c T \tau>0 \quad$, so

judge $\quad \frac{\partial D_{s}^{c}}{\partial \mu}=\frac{-A^{2} T \tau(1-\rho)(B-c T \tau)}{\left(A^{2}-2 T \mu \tau\right)^{2}}<0 \quad, \quad \frac{\partial D_{r}^{c}}{\partial \mu}=\frac{-A^{2} T \tau \rho(B-c T \tau)}{\left(A^{2}-2 T \mu \tau\right)^{2}}<0$, $\frac{\partial D_{s}^{d}}{\partial \mu}=\frac{A^{2} T \rho \tau(-B+c T \tau)}{\left(A^{2}-2 T \mu \tau(1+\rho)\right)^{2}}<0$, proposition 2 is proved.

Proposition 2 shows that under centralized and decentralized decision-making, the supplier of fresh agricultural products invests in fresh-keeping efforts. The optimal demand of fresh agricultural products online and off-line is negatively correlated with the cost coefficient of fresh-keeping, indicating that the demand of fresh agricultural products will be reduced with the increase of the cost coefficient of fresh-keeping. 
Proposition $3 \frac{\partial k^{d}}{\partial \mu}<0, \frac{\partial k^{s}}{\partial \mu}<0$

Proof: The formula 9and 16 are respectively obtained. : $\frac{\partial k^{c}}{\partial \mu}=\frac{-2 A T \tau(B-c T \tau)}{\left(A^{2}-2 T \mu \tau\right)^{2}}<0$,

$\frac{\partial k^{d}}{\partial \mu}=\frac{2 A T \tau(1+\rho)(-B+c T \tau)}{\left(A^{2}-2 T \mu \tau(1+\rho)\right)^{2}}<0$, so proposition 3 is proved.

Proposition 3 shows that whether under centralized or decentralized decision-making, the level of freshkeeping effort is negatively correlated with the coefficient of fresh-keeping cost, indicating that the coefficient of fresh-keeping cost is too large to improve the level of fresh-keeping effort.

Proposition 4 By comparing the two modes of centralized decision and decentralized decision, we can draw a conclusion that when $A^{2}-T \mu \tau<0, \quad p^{c}<p^{d}$; when $A^{2}-T \mu \tau>0, \quad p^{c}>p^{d}$ 。

Proof: From formula 6 and formula 12, we can calculate the difference in price. $\quad p^{c}-p^{d}=\frac{2 \mu \rho(B-c T \tau)\left(A^{2}-T \mu \tau\right)}{\left(A^{2}-2 T \mu \tau\right)\left(A^{2}-2 T \mu \tau(1+\rho)\right)} \quad, \quad$ from Theorem $\quad 1, \quad A^{2}-2 T \mu \tau<0$ and $c T \tau-B<0$, therefore When $A^{2}-T \mu \tau<0, p^{c}<p^{d}$; When $A^{2}-T \mu \tau>0, p^{c}>p^{d}$, so proposition 4 is proved.

Proposition 5 The total profit of the supply chain under centralized decision is greater than the total profit of supply chain under decentralized decision, $\pi^{d}<\pi^{c}$ 。

Proof: We can be obtained by Theorem 1: $2 T \mu \tau-A^{2}>0$, so we can easily get, $\pi^{d}-\pi_{s}^{d}-\pi_{r}^{d}=\frac{2 T^{2} \mu^{3} \rho^{2} \tau^{2}(B-c T \tau)^{2}}{\left(2 T \mu \tau-A^{2}\right)\left(A^{2}-2 T \mu \tau(1+\rho)\right)^{2}}>0$, so proposition 5 is proved.

From proposition 4 and 5 , it can be concluded that the product price under centralized decision is higher than that under decentralized decision, while the profit of supply chain system under centralized decision is greater than the total profit of decentralized decision. Because of the existence of double marginal effect, the profit of supply chain system under decentralized decision cannot reach the optimum, therefore, it is necessary to set up reasonable coordination strategy to achieve supply chain coordination as far as possible.

\section{4. "Fresh-Keeping Cost Sharing + Revenue Sharing" Coordination Contract}

Due to the existence of double marginal effects and the limitations of the level of fresh keeping efforts provided by supplier, in the decentralized supply chain system, the loss caused by inefficient decision-making of node members still occurs. In the face of the overall poor performance of the supply chain contract, scientific and reasonable can to some extent alleviate the double channel conflict.

In this section, the coordination contract of " fresh-keeping cost sharing and revenue sharing" is the fresh agricultural products supplier to share the proceeds of online retailer, including online retailer can share earnings ratio of $\varphi$; At the same time, fresh agricultural retailer need to share the cost of fresh-keeping efforts, of which the ratio of sharing the cost of fresh-keeping is $\eta$.

The profits of supplier for fresh agricultural products:

$$
\pi_{s}=(1-\varphi)(p-c) D_{s}+(w-c) D_{r}-(1-\eta) C(k)
$$

The profits of retailer for fresh agricultural products:

$$
\pi_{r}=\varphi(p-c) D_{s}+(p-w) D_{r}-\eta C(k)
$$

By solving the above equation, the following solution is gained:

$$
\begin{gathered}
k^{*}=\frac{A(T \tau(c-w \rho+c(-1+\rho) \varphi)+B(-1+\rho+\varphi-\rho \varphi))}{2 T(-1+\eta) \mu \tau+A^{2}(-1+\rho)(-1+\varphi)} \\
p^{*}=\frac{1}{2}\left(c-\frac{(c-w) \rho}{\rho+\varphi-\rho \varphi}+\frac{2 B(-1+\eta) \mu+A^{2}(c-w \rho+c(-1+\rho) \varphi)}{2 T(-1+\eta) \mu \tau+A^{2}(-1+\rho)(-1+\varphi)}\right)
\end{gathered}
$$

In order to make the optimal value under decentralized decision-making reach the optimal value under centralized decision-making, it is necessary to satisfy $p^{*}=p^{c}$ and $k^{*}=k^{c}$ under the coordination contract. 
At this time, the wholesale price $w$ is no longer a decision variable, but as a regulatory variable, the supply chain system achieves coordination.

Theorem 3 When the coordination contract $(w, \varphi, \eta)$ satisfies $w=c, \varphi=\frac{\eta-\rho}{1-\rho}$, and when fresh- keeping cost sharing ratio satisfies $\frac{2 T \mu \rho \tau\left(2 T \mu \tau-A^{2}\right)}{\left(A^{2}-2 T \mu(1+\rho) \tau\right)^{2}} \leq \eta \leq \frac{2 T \mu \rho \tau}{2 T \mu(1+\rho) \tau-A^{2}}$, the fresh agricultural product supply chain is coordinated.

Proof: By the above assumptions, it can draw $w=c$ and $\varphi=\frac{\eta-\rho}{1-\rho}$, take them into the profit function of fresh agricultural supplier and retailer, We can obtain profits from retailer and supplier, $\quad \pi_{s}^{*}=\frac{\mu(1-\eta)(B-c T \tau)^{2}}{2\left(2 T \tau \mu-A^{2}\right)} \quad, \quad \pi_{r}^{*}=\frac{\eta \mu(B-c T \tau)^{2}}{2\left(2 T \mu \tau-A^{2}\right)} \quad, \quad$ they $\quad$ satisfies $\pi_{s}^{*}+\pi_{r}^{*}=-\frac{\mu(B-c T \tau)^{2}}{2\left(A^{2}-2 T \mu \tau\right)}=\pi^{c}$, at this time, the optimal decision of fresh agricultural products supplier and retailer is equal to the optimal decision of supply chain system under centralized decision. In addition, it is also required to meet $\pi_{s}^{*} \geq \pi_{s}^{c}$ and $\pi_{r}^{*} \geq \pi_{r}^{c}$, by solving the above inequation, the following solution is gained:

$$
\frac{2 T \mu \rho \tau\left(2 T \mu \tau-A^{2}\right)}{\left(A^{2}-2 T \mu(1+\rho) \tau\right)^{2}} \leq \eta \leq \frac{2 T \mu \rho \tau}{2 T \mu(1+\rho) \tau-A^{2}} \text {, so theorem } 3 \text { is proved. }
$$

Theorem 3 shows that the contract model of "revenue sharing + fresh-keeping cost sharing" can coordinate the supply chain of fresh agricultural products. Compared with decentralized decision-making, retailer can control the ratio of fresh-keeping cost to improve the profit of supplier and retailer. In this contract model, although $w$ is an adjusting variable, it is still decided by fresh product supplier. Therefore, in real life, if supplier do not set the corresponding wholesale price, fresh product supply chain will not be able to achieve coordination.

\section{Numerical Analysis}

In order to correct and rationality of the relevant conclusions to verify the model, based on fresh agricultural products industry data to verify the optimal solution under different models, parameter values are shown in Table 2.

\begin{tabular}{c|c|c|c|c|c|c|c}
\multicolumn{1}{c}{ Table-2. parameters assignment table. } \\
\hline$\lambda_{0}$ & $\tau$ & $\theta_{0}$ & $T$ & $\beta$ & $\xi$ & $c$ & $\rho$ \\
\hline 0.9 & 300 & 1 & 20 & 2 & 0.2 & 1 & 0.4 \\
\hline
\end{tabular}

\subsection{The Impact of Fresh-Keeping Cost Coefficient $\mu$ on Decision Variables}

The Table 2 into the corresponding numerical decision variables, we can get the Figure $2-4$ by using MATLAB software. 


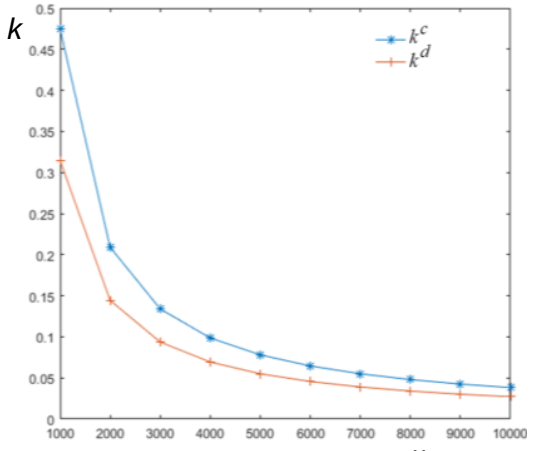

Fig-2. Effect of fresh-keep nig effort cost coefficient on $\mathrm{K}$

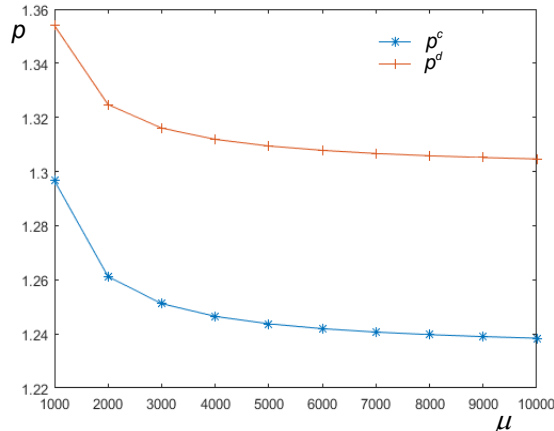

Fig-3. Effect of fresh-keep ing effort cost coefficient on $\mathrm{P}$

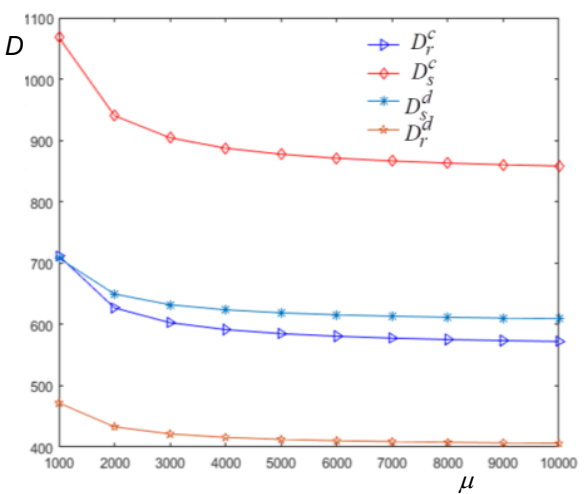

Fig-4. Effect of fresh-keep ing effort cost coefficient on D

Figure 2 shows that when $\mu>\frac{A^{2}}{2 T \tau(1+\rho)}$, with the increase of fresh-keeping cost coefficient, the level of fresh-keeping effort under centralized and decentralized decision-making is reduced, which verifies proposition 3.

Figure 3 shows that the increase of fresh-keeping cost coefficient will lead to the decrease of the optimal price of fresh agricultural products under both centralized and decentralized decision-making. The analysis shows that the reason why the supplier and retailer of fresh agricultural products adopt the low price is that with the increase of the fresh-keeping cost coefficient, they can not obtain the advantage by adopting the fresh-keeping decision-making. This verifies proposition 1.

Figure 4 shows that under centralized and decentralized decision-making, the increase of fresh-keeping cost coefficient will reduce the demand for fresh agricultural products, and no matter what decision-making model, online channel sales volume is always higher than offline channel sales. The comparison of the four curves in Figure 4 shows that the demand under centralized decision-making is higher than that under decentralized decision-making when the fresh-keeping cost coefficient changes, which verifies Proposition 2.

In conclusion, under the decentralized decision-making model, the overall profit of fresh agricultural products supply cannot reach the optimal system profit under the centralized decision-making, so we can design a certain coordination contract model for fresh agricultural products dual-channel supply chain.

\subsection{Numerical Example}

By substituting the parameter values of Table 2 into the relevant table functions and taking $\mu=2200$, the optimal decision-making of supplier and retailer of fresh agricultural products under centralized and decentralized decision-making can be obtained, as shown in Table 3. 
Table-3. Calculation results of different decision models.

\begin{tabular}{l|l|l|l}
\hline Optimal value & Centralized decision & Decentralization decision & comparison \\
\hline Optimal pricing & 1.26 & 1.32 & $p^{c}<p^{d}$ \\
\hline Wholesale price & - & 1.14 & - \\
\hline Supplier fresh-keeping & 0.188 & 0.13 & $k^{c}>k^{d}$ \\
\hline Online sales volume & 930.2 & 644.6 & $D_{s}^{c}>D_{s}^{d}$ \\
\hline Offline sales & 620.1 & 429.8 & $D_{r}^{c}>D_{r}^{d}$ \\
\hline Supplier profit & - & 250.7 & - \\
\hline Retailer profit & - & 77.0 & - \\
\hline Supply chain profit & 361.7 & 327.7 & $\pi^{c}>\pi^{d}$ \\
\hline
\end{tabular}

Table 3 shows that the reason why the profit of supply chain under decentralized mode is not optimal compared with centralized mode is that, in decentralized mode, the enthusiasm of supplier'fresh-keeping input is greatly affected, supplier reduce costs by reducing their fresh-keeping efforts, but because the market is sensitive to the freshness of fresh agricultural products. As a result, the two-channel sales are greatly reduced, and ultimately the total profit of fresh agricultural products in the two-channel supply chain system is reduced. Therefore, the model provides dual channel to fresh-keeping of fresh agricultural products on the supplier in the double marginal effect of decentralized mode of fresh agricultural products in the dual channel supply chain model, the two sides as well as the profits of the supply chain system have not reached the level of centralized mode, which verified the correctness of the conclusion of the former. follows:

Under the contract model of "revenue sharing + fresh-keeping cost sharing ", the profit of supplier is as

$$
\pi_{s}^{*}=\frac{\mu(1-\eta)(B-c T \tau)^{2}}{2\left(2 T \tau \mu-A^{2}\right)}=361.74(1-\eta)
$$

Under this contract model, supplier will have $361.74(1-\eta) \geq 250.7$, that is $\eta \leq 0.31$, to ensure that their profits are not affected. This means that if the profit of fresh agricultural products supplier is greater than that of supplier under decentralized decision-making, retailer are required to bear up to $31 \%$ of the cost of fresh-keeping effort, so that the optimal level of fresh-keeping effort can be raised to that under centralized decision-making. Strive for the best price and achieve the optimal price under centralized decision. At the same time, the profit of the retailer under this contract model is:

$$
\pi_{r}^{*}=\frac{\eta \mu(B-c T \tau)^{2}}{2\left(2 T \mu \tau-A^{2}\right)}=361.74 \eta
$$

This shows that if the retailer accepts the contract model, the retailer needs to consider its own profit higher than the profit under the decentralized decision-making. There is $361.74 \eta \geq 77.0$, that is $\eta \geq 0.21$, indicating that the retailer needs to bear at least $21 \%$ of the cost of preservation effort, which can make the fresh agricultural products supply chain to achieve coordination.

Therefore, in this coordination contract $\operatorname{model}(w, \varphi, \eta)$, the value of $\eta$ must satisfy $\eta \in[0.21,0.31]$, and the specific value $\eta$ also depends on the bargaining power between fresh produce suppliers and retailer.

\section{Conclusion}

In the past, the dual-channel supply chain of fresh agricultural products neglected the effect of fresh agricultural products fresh-keeping cost coefficient on profit. Under the condition of retailer's uniform pricing, this paper considers consumer utility and consumer's novelty to fresh agricultural products under the dualchannel supply chain system of fresh agricultural products composed of a single supplier and a single retailer. Under the background of freshness requirement, a dual-channel supply chain model is constructed. Stackelberg game method is used to solve the model. A coordinated approach of cost subsidy for fresh-keeping effort provided by dominant supplier is provided to improve the operational efficiency of the dual-channel supply chain for fresh agricultural products. Supplier share a certain proportion of retail insurance. Fresh cost effectively encourages retailer to increase the cost of retail channel fresh-keeping investment, making the sales volume of offline channels increased, and thus making the profits of both sides increased. The model analysis shows that, compared with the centralized model, the enthusiasm of the decentralized model will be reduced, resulting in the overall sales volume of fresh agricultural products in two channels will be reduced, which makes the overall supply chain efficiency reduced. The coordination model of "revenue sharing + fresh- 
keeping cost sharing" proposed by supplier can be fully coordinated. The post numerical analysis shows that the specific proportion can improve the profits of both sides and achieve coordination.

\section{Acknowledgement}

This research is supported by "Chinese Ministry of Education, Humanities and Social Science Project" under Grant No. 12YJA630185.

\section{References}

Aviv, Y., \& Pazgal, A. (2008). Optimal pricing of seasonal products in the presence of forward-looking consumers. Manufacturing \& Service Operations Management, 1O(3), 339-359.

Cai, X., Chen, J., \& Xiao, Y. (2010). Optimization and coordination of fresh product supply chains with freshness-keeping effort. Production $\mathscr{E}^{2}$ Operations Management, 19(3), 261-278.

Ferguson, M., \& Ketzenberg, M. E. (2006). Information sharing to improve retail product freshness of perishables. Production and Operations Management, 15(1), 57-73.

Gu, B., Fu, Y., \& Li, Y. (2018). Fresh-keeping effort and channel performance in a fresh product supply chain with lossaverse consumers' returns. Mathematical Problems in Engineering.

Huang, H., He, Y., \& Li, D. (2018). Pricing and inventory decisions in the food supply chain with production disruption and controllable deterioration. Journal of Cleaner Production, 180, 280-296.

Lee, Y. P., \& Dye, C. Y. (2012). An inventory model for deteriorating items under stock-dependent demand and controllable deterioration rate. Computers \& Industrial Engineering, 63(2), 474-482.

Martin, R. (2015). China must improve its cool supply chain to keep pace with demand for fresh food.

Nagurney, A., Besik, D., \& Yu, M. (2018). Dynamics of quality as a strategic variable in complex food supply chain network competition: The case of fresh produce. Chaos: An Interdisciplinary Journal of Nonlinear Science, 28(4), 043124 .

Su, X., \& Zhang, F. (2008). Strategic customer behavior, commitment, and supply chain performance. Management Science, $54(10), 1759-1773$

Tiaojun, X., Danqin, Y., \& Houcai, S. (2011). Coordinating a supply chain with a quality assurance policy via a revenuesharing contract. International Journal of Production Research, 49(1), 99-120.

Yu, M., \& Nagurney, A. (2013). Competitive food supply chain networks with application to fresh produce. European Journal of Operational Research, 224(2), 273-282. 